\title{
Hyperhomocysteinemia inhibits tibial fracture healing in rats through PI3K/AKT signaling pathway
}

\author{
SU LIU ${ }^{1}$, YUNZONG HUANG ${ }^{1}$, SHOUJING TIAN ${ }^{1}$, WEI ZHANG $^{1}$, YOUJIA XU ${ }^{2}$ and JIANFEI GE ${ }^{1}$ \\ ${ }^{1}$ Department of Orthopedics, The Affiliated Zhangjiagang Hospital of Soochow University, \\ Zhangjiagang, Jiangsu 215600; ${ }^{2}$ Department of Orthopedics,
} The Second Affiliated Hospital of Soochow University, Suzhou, Jiangsu 215004, P.R. China

Received September 16, 2019; Accepted November 11, 2019

DOI: $10.3892 /$ etm.2020.8412

\begin{abstract}
To explore the influence of hyperhomocysteinemia (hHcys) on the tibial fracture healing in rats and its effect on the phosphatidylinositol 3-hydroxy kinase $(\mathrm{PI} 3 \mathrm{~K}) /$ protein kinase B (AKT) signaling pathway. A total of 36 Sprague-Dawley rats were randomly divided into sham group $(n=12)$, tibial fracture group $(n=12)$ and hHcys + fracture group $(n=12)$. The rats in tibial fracture group underwent the tibial fracture surgery, while the model of tibial fracture and hHcys was established in hHcys + fracture group. The level of plasma homocysteine (Hcy) in each group was analyzed using the full-automatic biochemical analyzer, the fracture stress biomechanical measurement was performed, and the ultimate bending strength and torque were calculated. Moreover, the protein expressions of PI3K and phosphorylated (p)-AKT in tibial tissues were detected using western blotting, the messenger ribonucleic acid (mRNA) levels of $\mathrm{Bcl}-2$ associated $\mathrm{X}$ protein (Bax) and caspase-3 were detected using quantitative polymerase chain reaction (qPCR), the apoptosis was detected via terminal deoxynucleotidyl transferase-mediated dUTP nick end labeling (TUNEL) staining, and the expressions of inflammatory factors were detected via immunohistochemistry. Compared with sham group, tibial fracture group and hHcys + fracture group had a significantly increased level of plasma Hcy, significantly decreased ultimate bending strength and torque, obviously decreased relative protein expressions of PI3K and p-AKT, increased mRNA levels of Bax and caspase- 3 and an increased expression of pro-inflammatory factor tumor necrosis factor- $\alpha$ (TNF- $\alpha$ ). Compared with tibial fracture group, hHcys + fracture group had a higher level of plasma Hcy, lower ultimate bending strength and torque, lower relative protein expressions of PI3K and p-AKT, higher mRNA levels of Bax and caspase-3,
\end{abstract}

Correspondence to: Dr Jianfei Ge, Department of Orthopedics, The Affiliated Zhangjiagang Hospital of Soochow University, 68 Jiyang West Road, Zhangjiagang, Jiangsu 215600, P.R. China E-mail: dbfrq376@163.com

Key words: hyperhomocysteinemia, tibial fracture, PI3K/AKT signaling pathway, inflammatory response a higher apoptosis rate and a higher expression of TNF- $\alpha$. hHcys blocks the downstream apoptotic signal transduction, promotes apoptosis and inflammatory response, and affects fracture healing through affecting the PI3K/AKT signaling pathway.

\section{Introduction}

Tibial fracture is a clinically common type of long tubular bone fracture accounting for $13.7 \%$ of whole body fractures (1). The tibial fracture healing is a very complex process of injury repair, which can be affected by many factors. Hyperhomocysteinemia (hHcys) is an important risk factor for cardiovascular disease and thrombotic disease (2). The clinical manifestations of hHcys include developmental retardation and skeletal anomalies, and high-level homocysteine (Hcy) will damage the function of osteocytes regulating bone remodeling, thereby resulting in fractures. Hcy, through increasing osteoclast activity, can regulate bone remodeling (3), induce apoptosis of bone marrow stromal cells (4), osteocytes and osteoblasts $(5,6)$, and inhibit osteoblast differentiation (7).

The phosphatidylinositol 3-hydroxy kinase (PI3K)/protein kinase B (AKT) signal transduction pathway plays an important role in post-traumatic repair and healing, which is a regulatory pathway for cell growth. Studies have proved that this pathway is essential for the differentiation of osteoprogenitor cells $(8,9)$. Tetrahydroxystilbene glycoside (TSG) can facilitate the differentiation of MC3T3-E1 cells through activating the PI3K/AKT signal transduction. The downregulation of PTEN, a negative feedback regulator of PI3K/AKT in osteoblasts, can activate the PI3K/AKT signaling pathway, thus affecting osteocyte differentiation (10).

In this study, therefore, the effect of hHcys on the tibial fracture healing in rats was observed, and the role of PI3K/AKT signaling pathway in tibial fracture was explored, hoping to provide a theoretical basis for the diagnosis and treatment of tibial fracture.

\section{Materials and methods}

Animal experiments and grouping. A total of 36 specific pathogen-free male Sprague-Dawley rats weighing 200-240 g were adaptively fed in an environment in line with animal 
ethical requirements for 1 week before surgery, and they had free access to food and water. They were deprived of food, but not water, for $12 \mathrm{~h}$ before surgery, and divided into sham group $(n=12)$, tibial fracture group $(n=12)$ and hHcys + fracture group $(n=12)$ using a random number table. This study was approved by the Animal Ethics Committee of Soochow University Animal Center (Suzhou, China).

Modeling. i) Establishment of tibial fracture model (11): After intraperitoneal anesthesia with pentobarbital sodium, the left lower limb was depilated, and the fascia and muscle were separated to expose the tibia. Then the fracture model was established in the middle tibia, and fixed intramedullaryly using the Kirschner wire (1 $\mathrm{mm}$ in diameter). ii) Establishment of hHcys model (12): After establishment of tibial fracture model, the rats in hHcys + fracture group were fed with L-methionine for 4 weeks. iii) In sham group, the tibia was exposed only without establishing the tibial fracture model, and the rats were fed with normal diet.

Main instruments and equipment. AG-1S electronic universal mechanical testing machine (Shimadzu), PI3K, p-AKT, Bax, caspase-3 and tumor necrosis factor- $\alpha$ (TNF- $\alpha$ ) primary antibodies (Abcam), terminal deoxynucleotidyl transferase-mediated dUTP nick end labeling (TUNEL) assay kit (Shanghai Beyotime Biotechnology), quantitative polymerase chain reaction (qPCR) kit (Vazyme), immunohistochemistry kit (Maxim), bicinchoninic acid (BCA) protein quantification kit (Shanghai Beyotime Biotechnology), fluorescence qPCR instrument (ABI 7500; Applied Biosystems; Thermo Fisher Scientific, Inc.), Image-Lab image analysis system (Bio-Rad Laboratories), and Leica DM4000B LED microscope (Leica Microsystems $\mathrm{GmbH}$ ).

Detection of plasma Hcy concentration. The venous blood was drawn, placed in the test tube containing ethylenediaminetetraacetic acid (EDTA) and coagulant, and centrifuged at $2,000 \times \mathrm{g}$ at $4^{\circ} \mathrm{C}$ for $5 \mathrm{~min}$ to separate the plasma and serum. Then the plasma Hcy concentration was measured using a full-automatic biochemical analyzer. Plasma (100-150 $\mu \mathrm{l})$ was used for each measurement, and each measurement was repeated for 3 times.

Biomechanical measurement of fracture stress. After the rats were sacrificed, the tibial specimens were taken for the biomechanical three-point bending test at a loading rate of $0.01 \mathrm{~mm} / \mathrm{sec}$ and span of $15 \mathrm{~mm}$. The mechanical properties of fractured tibia were analyzed using a clinical biomechanical three-point bending strain detector, and the load-displacement relation curve and stress-strain relation curve were plotted, based on which the ultimate bending strength and torque were read and calculated.

Determination of protein expressions of P13K and $p$-AKT in tibial tissues via western blotting. The tibial tissues were taken, fully lysed with cell lysis buffer in an ultrasonic homogenizer and centrifuged at $3,500 \mathrm{xg}$ at $4^{\circ} \mathrm{C}$ for $10 \mathrm{~min}$. After the supernatant was discarded, the protein samples were obtained, and the total protein concentration was determined using the BCA protein concentration kit. The protein was separated via gel electrophoresis, transferred onto a polyvinylidene fluoride (PVDF) membranes (Roche Diagnostics), sealed at room temperature and incubated with $\mathrm{PI} 3 \mathrm{~K}$ and $\mathrm{p}-\mathrm{AKT}$ primary antibodies at $37^{\circ} \mathrm{C}$ for $1 \mathrm{~h}$, with $\beta$-actin antibody as an internal reference. After the membrane was washed, the protein was incubated again with horse radish peroxidase (HRP)-labeled secondary antibodies, and the membrane was washed again. Finally, the image was developed using the electrochemiluminescence (ECL) kit in a darkroom.

Determination of messenger ribonucleic acid (mRNA) levels of Bax and caspase-3 via qPCR. The total RNA was extracted from tibial tissues using the TRIzol method (Invitrogen; Thermo Fisher Scientific, Inc.), and its concentration was measured using a spectrophotometer. Then the total RNA was reversely transcribed into complementary deoxyribose nucleic acid (cDNA) and quantitatively amplified using the reverse transcription kit and qPCR kit. The amplification conditions are as follows: pre-denaturation at $95^{\circ} \mathrm{C}$ for $1 \mathrm{~min}$, denaturation at $95^{\circ} \mathrm{C}$ for $5 \mathrm{sec}$, and annealing/extension at $58^{\circ} \mathrm{C}$ for $15 \mathrm{sec}$, a total of 40 cycles. Primer sequences of glyceraldheyde 3-phosphate dehydrogenase (GAPDH) (internal reference gene), forward, 5'-GGTGCTGAGTATGTCGTGGA-3' and reverse, 5'-TGCTGA CAATCTTGAGGGAG-3'. Primer sequences of caspase-3, forward, 5'-GACCCGGTGCCTCAGGATGC-3' and reverse, 5'-GTGGCATGAGCTCTTGATAATG-3'. Primer sequences of Bax forward, 5'-CAGAGGCGGGGGATGATTG-3' and reverse, 5'-TGTCCAGCCCATGATGGTTC-3'. The relative mRNA expression level was calculated using the $2^{-\Delta \mathrm{Ct}}$ formula.

Detection of apoptosis using TUNEL staining. The tissue sections were deparaffinized with xylene, dehydrated with gradient alcohol, and subjected to citrate antigen retrieval, followed by staining using the TUNEL staining kit. Then the sections were sealed with anti-fluorescence quenching blocking buffer and observed under a fluorescence microscope, and the number of positive cells and apoptosis rate were calculated based on apoptosis rate $=$ number of positive cells/total number of cells x $100 \%$.

Detection of expressions of inflammatory factors through immunohistochemistry. The tibial tissues were taken in each group, washed with phosphate-buffered saline (PBS), and decalcified with $10 \%$ EDTA decalcifying solution until softening of tissues, followed by paraffin embedding into $5 \mu \mathrm{m}$-thick coronal sections. Then the sections were deparaffinized with xylene, dehydrated with gradient alcohol, and subjected to citrate antigen retrieval, followed by staining using the immunohistochemistry kit. The sections were incubated with $3 \% \mathrm{H}_{2} \mathrm{O}_{2}$ at room temperature for $10 \mathrm{~min}$, washed, sealed with $5 \%$ goat serum at room temperature for $10 \mathrm{~min}$, and dropwise added with TNF- $\alpha$ primary antibody in a wet box at $4^{\circ} \mathrm{C}$ overnight. After rewarming at $37^{\circ} \mathrm{C}$, the sections were incubated again with biotinylated secondary antibody at room temperature for $10 \mathrm{~min}$, followed by color development using the diaminobenzidine (DAB) developer and counterstaining with hematoxylin. Finally, the absorbance was analyzed using the Motic Med 6.0 pathologic image analysis system.

Statistical analysis. Statistical Product and Service Solutions (SPSS) 24.0 (IBM Corp.) software was used for the data 
Table I. Comparison of fracture biomechanics in each group (mean $\pm \mathrm{SD})$.

\begin{tabular}{llcr}
\hline Group & $\mathrm{n}$ & Ultimate bending strength $(\mathrm{N})$ & Torque $(\mathrm{N} \cdot \mathrm{mm})$ \\
\hline Sham group & 6 & $116.78 \pm 12.65$ & $357.49 \pm 7.65$ \\
Tibial fracture group & 6 & $77.23 \pm 6.77^{\mathrm{a}}$ & $216.82 \pm 17.71^{\mathrm{a}}$ \\
hHcys + fracture group & 6 & $45.84 \pm 4.32^{\mathrm{a}, \mathrm{b}}$ & $124.15 \pm 11.84^{\mathrm{a}, \mathrm{b}}$
\end{tabular}

${ }^{\mathrm{a}} \mathrm{P}<0.05$ vs. sham group; ${ }^{\mathrm{b}} \mathrm{P}<0.05$ vs. tibial fracture group. hHcys, hyperhomocysteinemia.

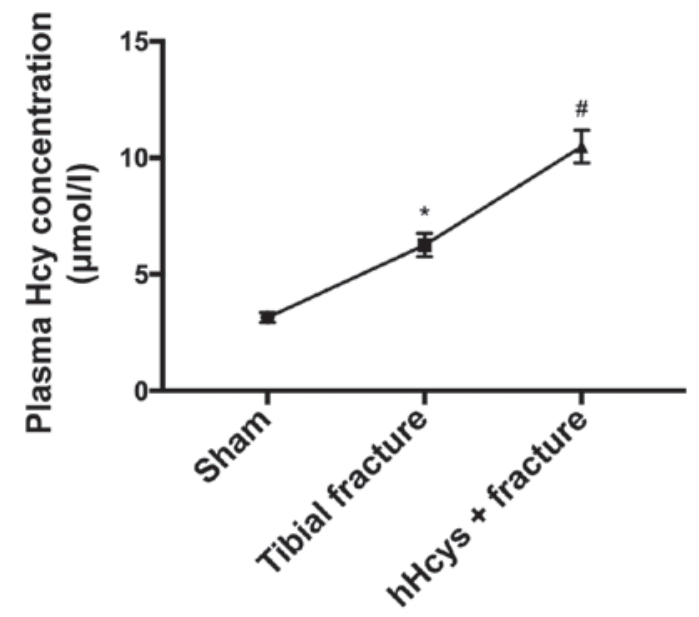

Figure 1. Comparison of plasma Hcy concentration in each group. ${ }^{*} \mathrm{P}<0.05$ vs. sham group; ${ }^{*} \mathrm{P}<0.05$ vs. tibial fracture group. Hcy, homocysteine.

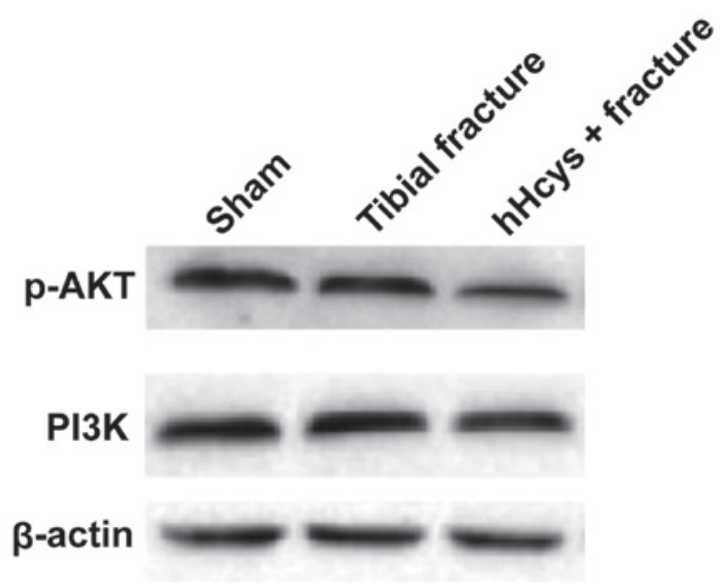

Figure 2. Relative protein expressions in tibial tissues in each group.

processing. The t-test was used for analyzing measurement data. Differences between two groups were analyzed by using the Student's t-test. Comparison between multiple groups was done using One-way ANOVA test followed by Post Hoc Test (Least Significant Difference). $\mathrm{P}<0.05$ suggested that the difference was statistically significant.

\section{Results}

Comparison of plasma Hcy concentration in each group. The plasma Hcy concentration was significantly increased in tibial

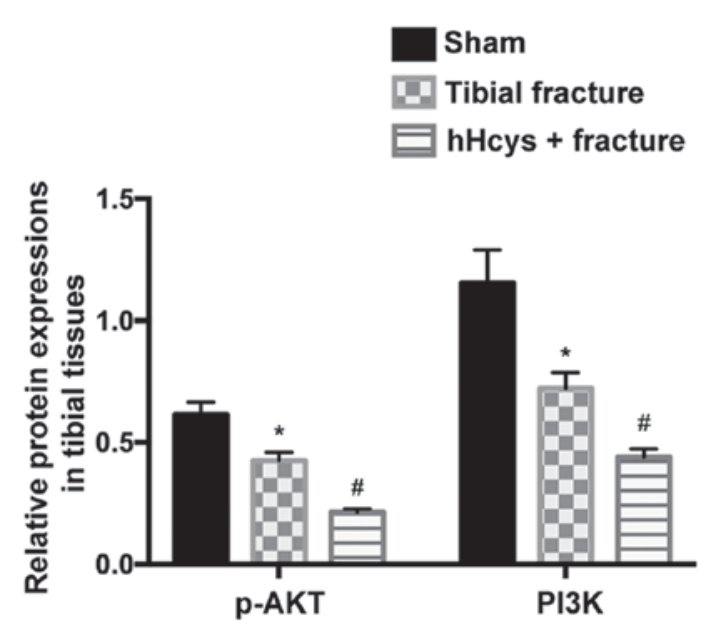

Figure 3. Comparison of relative protein expressions in tibial tissues in each group. ${ }^{\text {}} \mathrm{P}<0.05$ vs. sham group; ${ }^{"} \mathrm{P}<0.05$ vs. tibial fracture group.

fracture group and hHcys + fracture group compared with that in sham group $(\mathrm{P}<0.05)$, while it was also significantly increased in hHcys + fracture group compared with that in tibial fracture group $(\mathrm{P}<0.05)$, showing statistically significant differences (Fig. 1).

Comparison of fracture biomechanics in each group. The ultimate bending strength and torque were obviously decreased in tibial fracture group and hHcys + fracture group compared with those in sham group $(\mathrm{P}<0.05)$, while they declined in hHcys + fracture group compared with those in tibial fracture group $(\mathrm{P}<0.05)$, showing statistically significant differences (Table I).

Protein expressions of PI3K and $p$-AKT in tibial tissues detected via western blotting. The relative protein expressions of PI3K and p-AKT evidently declined in tibial fracture group and hHcys + fracture group compared with those in sham group $(\mathrm{P}<0.05)$, while they also declined in hHcys + fracture group compared with those in tibial fracture group $(\mathrm{P}<0.05)$, displaying statistically significant differences (Figs. 2 and 3).

Comparison of mRNA levels of Bax and caspase-3. Compared with sham group, tibial fracture group and hHcys + fracture group had increased mRNA levels of Bax and caspase-3 $(\mathrm{P}<0.05)$. The mRNA levels of Bax and caspase-3 were higher in hHcys + fracture group than those in hHcys + fracture group $(\mathrm{P}<0.05)$, and the differences were statistically significant (Fig. 4). 


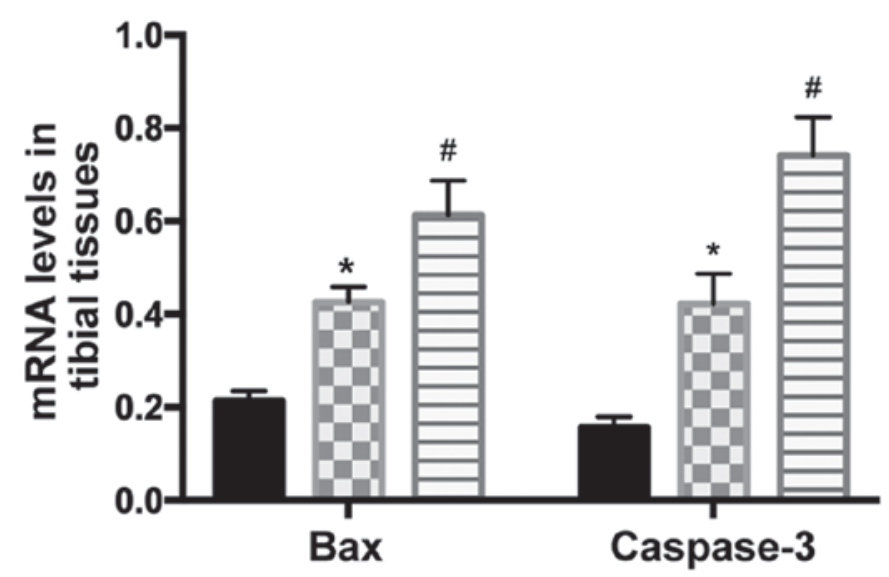

Sham

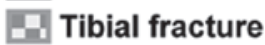

hHcys + fracture

Figure 4. Comparison of mRNA levels of Bax and caspase-3. ${ }^{*} \mathrm{P}<0.05$ vs. sham group; ${ }^{\text {"}} \mathrm{P}<0.05$ vs. tibial fracture group.
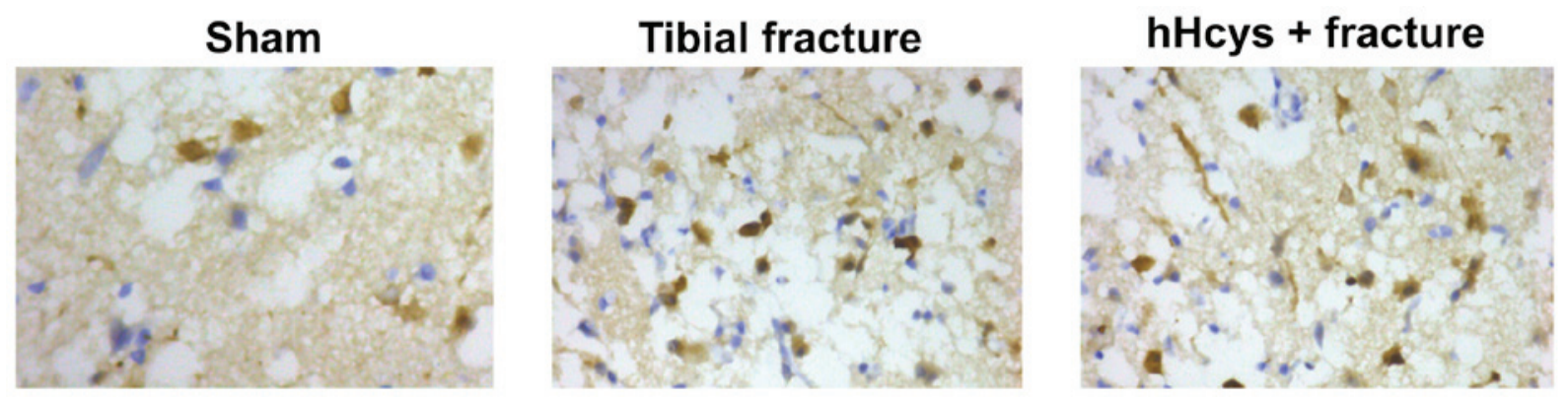

Figure 5. Apoptotic cells in tibial tissues in each group (TUNEL, $\mathrm{x} 400$ ).

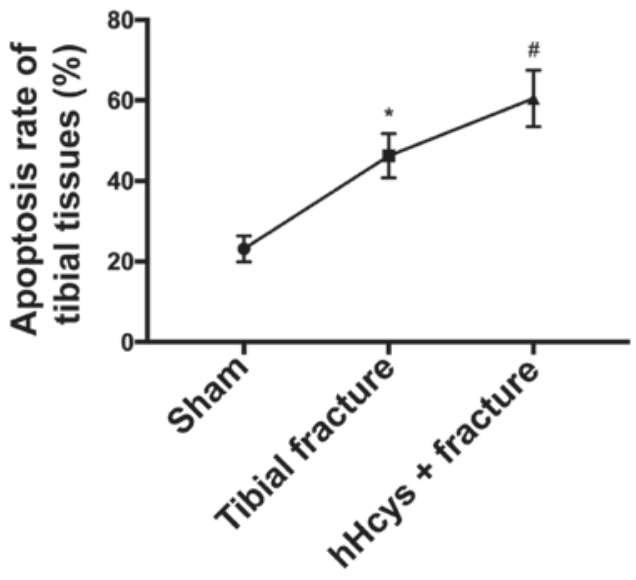

Figure 6. Comparison of apoptosis rate of tibial tissues in each group. "P $<0.05$ vs. sham group; ${ }^{~} \mathrm{P}<0.05$ vs. tibial fracture group.

Comparison of apoptosis rate. Tibial fracture group and hHcys + fracture group had a higher apoptosis rate than sham group $(\mathrm{P}<0.05)$, while hHcys + fracture group also had a higher apoptosis rate than tibial fracture group $(\mathrm{P}<0.05)$, and there were statistically significant differences (Figs. 5 and 6).

Comparison of expression of pro-inflammatory factor. The expression of TNF- $\alpha$ was increased in tibial fracture group and hHcys + fracture group compared with that in sham group $(\mathrm{P}<0.05)$, while it was also increased in hHcys + fracture group compared with that in tibial fracture group $(\mathrm{P}<0.05)$, and the differences were statistically significant (Figs. 7 and 8).

\section{Discussion}

The bone possesses a strong self-repairing ability, but $5-10 \%$ of fracture patients suffer from inadequate union, delayed union or nonunion. In this study, the plasma Hcy level rose after tibial fracture. Hcy is a kind of non-protein amino acid synthesized by methionine and recycled into methionine or converted into cysteine with the help of B vitamins. The increased level of plasma Hcy is associated with the increase in incidence rate of fracture, and the high-level plasma Hcy may affect bone health, which will lead to bone resorption disorders through stimulating osteoclast activity, and interfere with collagen cross-linking. The in vitro studies have shown that the elevated concentration of Hcy has an inhibitory effect on bone formation, which induces apoptosis through ROS-mediated mitochondrial pathway and NF- $\mathrm{KB}$ activation in human bone marrow mesenchymal stem cells (hBMSCs), and Hcy can promote the development of osteoporosis by reducing bone formation (13). Moreover, high-concentration Hcy inhibits the activity of lysyl oxidase (an enzyme involved in collagen cross-linking), and the interference with cross-linking will alter the bone matrix and result in fragile bones. Excessive Hcy also leads to loss of bone substance. Type I collagen, the main organic component in bone, is composed of two non-helical telopeptides and a triple helix region at the amino terminal $(\mathrm{N})$ and carboxy terminal (C) of the molecule, which stabilizes 

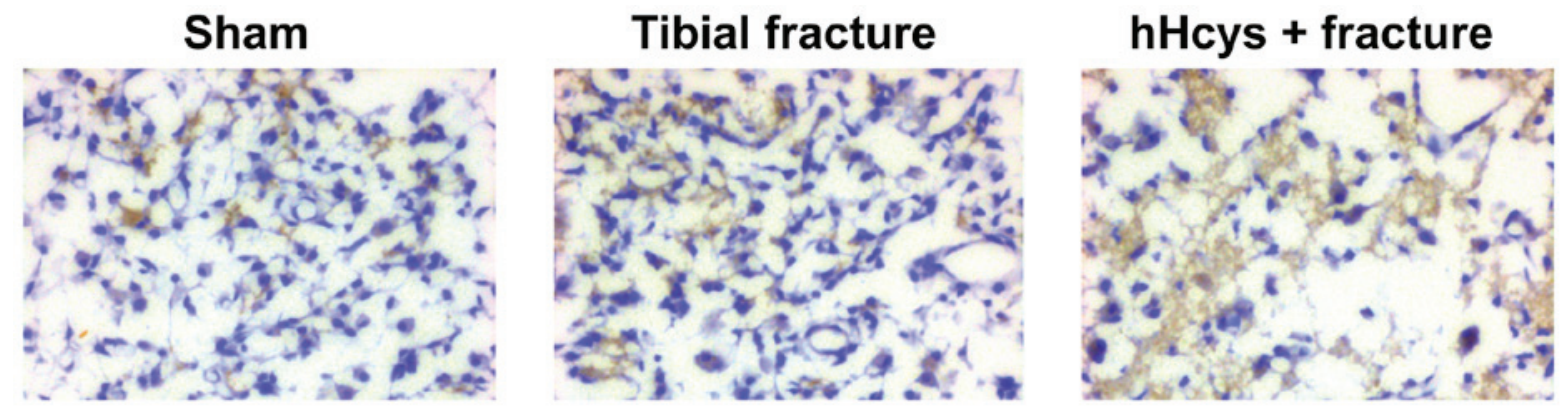

Figure 7. Expression of pro-inflammatory factor in tibial tissues in each group (immunohistochemistry, $\mathrm{x} 400$ ).

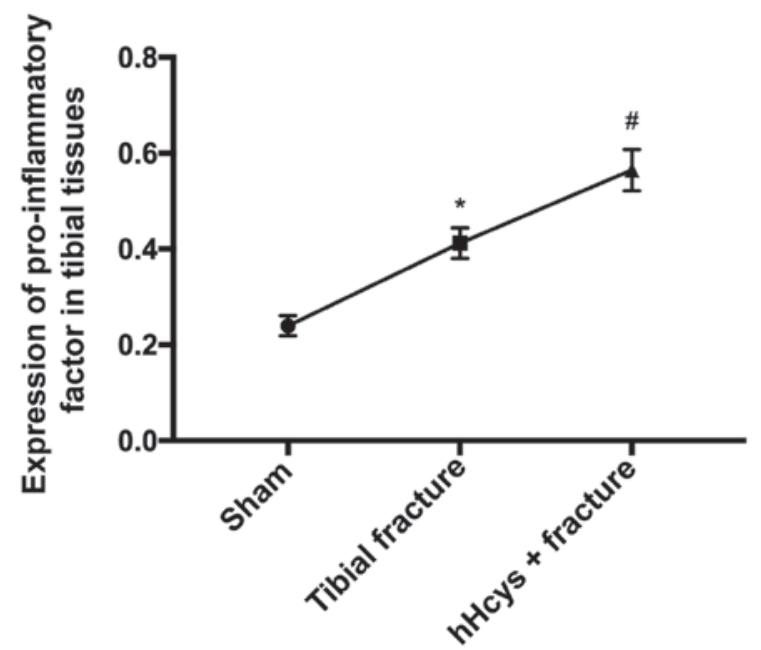

Figure 8. Comparison of expression of pro-inflammatory factor in tibial tissues in each group. ${ }^{*} \mathrm{P}<0.05$ vs. sham group; ${ }^{\#} \mathrm{P}<0.05$ vs. tibial fracture group.

the newly secreted collagen molecules through forming cross-linking between adjacent collagen molecules, thus affecting the tensile strength and toughness of bone $(14,15)$. In this study, it was found via biomechanical measurement that both ultimate bending strength and torque declined in hHcys + fracture group compared with those in tibial fracture group, indicating that excessive Hcy affects the bone quality in tibial fracture.

The bone is a kind of dynamic tissue that constantly renews the osteoclast activity through muscles, and absorbs mineralized bones and osteoblasts, forming the new bone matrix. During the whole process, some osteoblasts are embedded in new bones and differentiate into osteoblasts (16). The PI3K/AKT signaling pathway is an important pathway in stress fracture repair, and it is activated in stress fracture callus tissues, which is important for osteoblast differentiation and function $(17,18)$. The AKT signal regulates the osteogenesis after injury through producing osteoprotegerin, and inhibiting the AKT signal transduction in the rat model of fracture nonunion can reduce the transplantation and differentiation of MSCs into fracture callus tissues (19). In addition, studies have demonstrated that PI3K can regulate periosteal thickening in the early stage of fracture repair (20). Therefore, PI3K/AKT is an important signal transduction pathway for bone regeneration. In this study, the PI3K/AKT signaling pathway was damaged in rats after tibial fracture, and the expressions of PI3K and p-AKT obviously declined in hHcys + fracture group compared with those in tibial fracture group. Besides, the PI3K/AKT signaling pathway mediated apoptosis and inflammatory response, and affected osteocyte differentiation.

In conclusion, in the present study, the role of the PI3K/AKT signaling pathway in fracture repair was explored using the rat model of hHcys and tibial fracture. The results demonstrate that hHcys blocks the downstream apoptotic signal transduction, promotes apoptosis and inflammatory response, and affects fracture healing through affecting the PI3K/AKT signaling pathway.

\section{Acknowledgements}

Not applicable.

\section{Funding}

No funding was received.

\section{Availability of data and materials}

All data generated or analyzed during this study are included in this published article.

\section{Authors' contributions}

SL and JG designed the study and performed the experiments, SL and YH established the animal models, JG and ST collected the data, WZ and YX analyzed the data, SL and JG prepared the manuscript. All authors read and approved the final manuscript.

\section{Ethics approval and consent to participate}

This study was approved by the Animal Ethics Committee of Soochow University Animal Center (Suzhou, China).

\section{Patient consent for publication}

Not applicable.

\section{Competing interests}

The authors declare that they have no competing interests. 


\section{References}

1. Liu ZC, Xu YL, Jiang Y, Liu Y, Wei ZC, Liu SG and Yang SJ: Low-expression of IncRNA-ANCR promotes tibial fracture healing via targeting RUNX2. Eur Rev Med Pharmacol Sci 23 (Suppl 3): 60-66, 2019.

2. Stepanova TV, Ivanov AN, Tereshkina NE, Popyhova EB and Lagutina DD: Markers of endothelial dysfunction: Pathogenetic role and diagnostic significance. Klin Lab Diagn 64: 34-41, 2019 (In Chinese).

3. Behera J, George AK, Voor MJ, Tyagi SC and Tyagi N: Hydrogen sulfide epigenetically mitigates bone loss through OPG/RANKL regulation during hyperhomocysteinemia in mice. Bone 114: 90-108, 2018.

4. Cai B, Li X, Wang Y, Liu Y, Yang F, Chen H, Yin K, Tan X, Zhu J, Pan Z, et al: Apoptosis of bone marrow mesenchymal stem cells caused by homocysteine via activating JNK signal. PLoS One 8: e63561, 2013.

5. Takeno A, Kanazawa I, Tanaka K, Notsu M, Yokomoto M, Yamaguchi T and Sugimoto T: Activation of AMP-activated protein kinase protects against homocysteine-induced apoptosis of osteocytic MLO-Y4 cells by regulating the expressions of NADPH oxidase 1 (Nox1) and Nox2. Bone 77: 135-141, 2015.

6. Kanazawa I, Tomita T, Miyazaki S, Ozawa E, Yamamoto LA and Sugimoto T: Bazedoxifene ameliorates homocysteine-induced apoptosis and accumulation of advanced glycation end products by reducing oxidative stress in MC3T3-E1 cells. Calcif Tissue Int 100: 286-297, 2017.

7. Thaler R, Zwerina J, Rumpler M, Spitzer S, Gamsjaeger S, Paschalis EP, Klaushofer K and Varga F: Homocysteine induces serum amyloid A3 in osteoblasts via unlocking RGD-motifs in collagen. FASEB J 27: 446-463, 2013.

8. Fan YS, Li Q, Hamdan N, Bian YF, Zhuang S, Fan K and Liu ZJ: Tetrahydroxystilbene glucoside regulates proliferation, differentiation, and OPG/RANKL/M-CSF expression in MC3T3-E1 cells via the PI3K/Akt pathway. Molecules 23: 2306, 2018.

9. Chai C, Song LJ, Han SY, Li XQ and Li M: MicroRNA-21 promotes glioma cell proliferation and inhibits senescence and apoptosis by targeting SPRY1 via the PTEN/PI3K/AKT signaling pathway. CNS Neurosci Ther 24: 369-380, 2018.

10. Jing X, Cheng W, Wang S, Li P and He L: Resveratrol induces cell cycle arrest in human gastric cancer MGC803 cells via the PTEN-regulated PI3K/Akt signaling pathway. Oncol Rep 35: 472-478, 2016
11. Handool KO, Ibrahim SM, Kaka U, Omar MA, Abu J, Yusoff MS and Yusof LM: Optimization of a closed rat tibial fracture model. J Exp Orthop 5: 13, 2018

12. Chaouad B, Moudilou EN, Ghoul A, Zerrouk F, Moulahoum A, Othmani-Mecif K, Cherifi MEH, Exbrayat JM and Benazzoug Y: Hyperhomocysteinemia and myocardial remodeling in the sand rat, Psammomys obesus. Acta Histochem 121: 823-832, 2019.

13. Kim DJ, Koh JM, Lee O, Kim NJ, Lee YS, Kim YS, Park JY, Lee KU and Kim GS: Homocysteine enhances apoptosis in human bone marrow stromal cells. Bone 39: 582-590, 2006.

14. Thaler R,Agsten M, Spitzer S,Paschalis EP, Karlic H, Klaushofer K and Varga F: Homocysteine suppresses the expression of the collagen cross-linker lysyl oxidase involving IL-6, Fli1, and epigenetic DNA methylation. J Biol Chem 286: 5578-5588, 2011.

15. Saito M,Fujii K and Marumo K: Degree of mineralization-related collagen crosslinking in the femoral neck cancellous bone in cases of hip fracture and controls. Calcif Tissue Int 79: 160-168, 2006.

16. Boyce BF, Li J, Xing L and Yao Z: Bone Remodeling and the Role of TRAF3 in Osteoclastic Bone Resorption. Front Immunol 9: 2263, 2018 .

17. Yang A, Lu Y, Xing J, Li Z, Yin X, Dou C, Dong S, Luo F, Xie Z, Hou T, et al: IL-8 enhances therapeutic effects of BMSCs on bone regeneration via CXCR2-mediated PI3k/Akt signaling pathway. Cell Physiol Biochem 48: 361-370, 2018.

18. Ayala-Peña VB, Scolaro LA and Santillán GE: ATP and UTP stimulate bone morphogenetic protein-2,-4 and -5 gene expression and mineralization by rat primary osteoblasts involving PI3K/AKT pathway. Exp Cell Res 319: 2028-2036, 2013.

19. Qu Z, Guo S, Fang G, Cui Z and Liu Y: AKT pathway affects bone regeneration in nonunion treated with umbilical cord-derived mesenchymal stem cells. Cell Biochem Biophys 71: 1543-1551, 2015.

20. Scanlon V, Walia B, Yu J, Hansen M, Drissi H, Maye P and Sanjay A: Loss of Cbl-PI3K interaction modulates the periosteal response to fracture by enhancing osteogenic commitment and differentiation. Bone 95: 124-135, 2017.

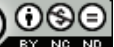

This work is licensed under a Creative Commons Attribution-NonCommercial-NoDerivatives 4.0 International (CC BY-NC-ND 4.0) License. 